\title{
M/M/1 Retrial Queueing System with Two Types of Vacation Policies under Erlang - K Type Service
}

\author{
G.AYYAPPAN \\ PONDICHERRY ENGINEERING \\ COLLEGE \\ PONDICHERRY,INDIA
}

\author{
GOPAL SEKAR \\ TAGORE ARTS COLLEGE \\ LAWSPET \\ PONDICHERRY,INDIA
}

\author{
A.MUTHU GANAPATHI \\ SUBRAMANIAN \\ TAGORE ARTS COLLEGE \\ PONDICHERRY,INDIA
}

\begin{abstract}
Consider a single server retrial queueing system in which customers arrive in a Poisson process with arrival rate $\lambda$ that which follows a Poisson process. Let $\mathrm{k}$ be the number of phases in the service station. The service time has Erlang k-type distribution with service rate $k \mu$ for each phase. Two types of vacation policies are discussed in this research paper that is Bernoulli type vacation and exhaustive type vacation. The vacation rate follows an exponential distribution with parameter $\boldsymbol{\alpha}$. We assume that the services in all phases are independent and identical and only one customer at a time is in the service mechanism. If the server is free at the time of a primary call arrival, the arriving call begins to be served in Phase 1 immediately by the server then progresses through the remaining phases and must complete the last phase and leave the system before the next customer enters the first phase. If the server is busy or on vacation, then the arriving customer goes to orbit and becomes a source of repeated calls. This pool of sources of repeated calls may be viewed as a sort of queue. Every such source produces a Poisson process of repeated calls with intensity $\boldsymbol{\sigma}$. If an incoming repeated call finds the server free, it is served in the same manner and leaves the system after service, while the source which produced this repeated call disappears. Otherwise, the system state does not change. We assume that the access from orbit to the service facility is governed by the classical retrial policy. This model is solved by using Matrix geometric technique. Numerical study have been done for Analysis of Mean number of customers in the orbit (MNCO), Truncation level (OCUT), Probabilities of server free, busy and in vacation for various values of $\boldsymbol{\lambda}, \boldsymbol{\mu}, \mathbf{k}, \mathbf{p}, \boldsymbol{\alpha}$ and $\boldsymbol{\sigma}$ in elaborate manner and also various particular cases of this model have been discussed.
\end{abstract}

\section{Keywords}

Single Server, Erlang k-type service, Bernoulli vacation, Matrix geometric method, exhaustive vacation, classical retrial policy.

\section{INTRODUCTION}

Queuing systems in which arriving customers who find all servers and waiting positions (if any) occupied may retry for service after a period of time are called Retrial queues [1, 2, 5, 6] Because of the complexity of the retrial queueing models, analytic results are

generally difficult to obtain. There are a great number of numerical and approximations methods available. In this paper we will place more emphasis on the solutions by Matrix geometric method [8]. In the literature, the analysis for queueing systems with vacations has been discussed through a considerable amount of work in recent years. Queues with server vacations occur in many engineering systems such as data switching systems, computer communication networks and telecommunication systems. Doshi $[\mathbf{3}, \mathbf{4}]$ has recorded prior work on vacation models and their applications in his survey paper. Keilson \& Servi [7] have introduced a queueing system with Bernoulli vacation scheduling service that is clearly applicable to queueing systems involving communication systems. Many examples such as production system, bank services, computer and communication networks, etc., work with different vacation policies. In this work we study retrial queuing system with two types of vacation policies namely Bernoulli and Exhaustive type of vacations under Erlang - $\mathrm{k}$ type service by Matrix Geometric Method.

\section{MODEL I}

\subsection{MODEL DESCRIPTION}

Consider a single server retrial queueing system with Bernoulli type vacation scheduling introduced by Keilson \& Servi [7] in which customers arrive in a Poisson process with arrival rate $\lambda$. These customers are identified as primary calls. Let $\mathrm{k}$ be the number of phases in the service station. Assume that the service time has Erlang-k distribution with service rate $k \mu$ for each phase. The vacation rate follows an exponential distribution with parameter $\boldsymbol{\alpha}$.

If the server is free at the time of a primary or repeated call arrival, then this arriving call begins to be served immediately and leaves the system after completion of the service. After completion of each service, the server has an option to go on vacation with probability $\mathbf{p}$ or continue to serve with probability (1-p). This type of vacation in queueing theory is called Single vacation with Bernoulli schedule. The single vacation means after completion of vacation period he can once again go for vacation after completing atleast one service. The server may return from the vacation at any time and is independent of number of customers in the system.

We assume that the services in all phases are independent and identical and only one customer at a time is in the service mechanism. If the server is free at the time of a primary call arrival, the arriving call begins to be served in Phase 1 immediately by the server then progresses through the remaining phases and must complete the last phase and leave the system before the next customer enters the first phase. If the server is busy or on vacation, then the arriving customer goes to orbit and becomes a source of repeated calls. This pool of sources of repeated calls may be viewed as a sort of queue. Every such source produces a Poisson process of repeated calls with intensity 
$\boldsymbol{\sigma}$. If an incoming repeated call finds the server free, it is served in the same manner and leaves the system after service, while the source which produced this repeated call disappears. Otherwise, the system state does not change.

We assume that the access from the orbit to the service facility follows the exponential distribution with rate $\boldsymbol{\sigma}$ which may depend on the current number $n,(n \geq 0)$ the number of customers in the orbit. That is, the probability of repeated attempt during the interval $(t, t+\Delta \mathrm{t})$, given that there are $\mathrm{n}$ customers in the orbit at time $t$ is $\mathbf{n} \boldsymbol{\sigma} \Delta \mathbf{t}$. It is called the classical retrial rate policy. The input flow of primary calls, interval between repetitions and service time in phases are mutually independent.

\subsection{MATRIX GEOMETRIC SOLUTIONS}

Let $\mathrm{N}(\mathrm{t})$ be the random variable which represents the number of customers in orbit at any time $t$ and $S(t)$ be the random variable which represents the phase in which customer is getting the service at time $t$. The random process is described as

$\{\langle\mathrm{N}(\mathrm{t}), \mathrm{S}(\mathrm{t})\rangle / \mathrm{N}(\mathrm{t})=0,1,2,3 \ldots ; \mathrm{S}(\mathrm{t})=0,1,2,3, \ldots, \mathrm{k}, \mathrm{k}+1\}$

$\mathrm{S}(\mathrm{t})=0$ if the server being idle

$\mathrm{S}(\mathrm{t})=\mathrm{i}$ for server being busy with the customer in the $\mathrm{i}^{\mathrm{th}}$

phase for $\mathrm{i}=1,2,3, \ldots \mathrm{k}$

$\mathrm{S}(\mathrm{t})=\mathrm{k}+1$ for the server to be on vacation.

The possible state spaces for single server retrial queueing with Erlang $\mathrm{K}$ - phases service are

$\{(\mathbf{i}, \mathbf{j}) / \mathrm{i}=\mathbf{0}, \mathbf{1}, \mathbf{2}, 3, \ldots ; \mathrm{j}=\mathbf{0}, \mathbf{1}, \mathbf{2}, 3, \ldots, \mathrm{k}, \mathrm{k}+\mathbf{1}\}$

The infinitesimal generator matrix $\mathbf{Q}$ for this model is given below

$Q=\left(\begin{array}{llllll}\mathbf{A}_{00} & \mathbf{A}_{0} & \mathbf{O} & \mathbf{O} & \mathbf{O} & \ldots \\ \mathbf{A}_{10} & \mathbf{A}_{11} & \mathbf{A}_{0} & \mathbf{O} & \mathbf{O} & \ldots \\ \mathbf{O} & \mathbf{A}_{21} & \mathbf{A}_{22} & \mathbf{A}_{0} & \mathbf{O} & \ldots \\ \mathbf{O} & \mathbf{O} & \mathbf{A}_{32} & \mathbf{A}_{33} & \mathbf{A}_{0} & \cdots\end{array}\right)$

The matrices $\mathbf{A}_{00}, \mathbf{A}_{\mathbf{0 1}}, \mathbf{A}_{\mathbf{n} \mathbf{n}-1}, \mathbf{A}_{\mathbf{n} \mathbf{n}}$ and $\mathbf{A}_{\mathbf{n} \mathbf{n} \mathbf{1}}$ for $\mathrm{n}$ $=1,2,3, \ldots$ in the infinitesimal matrix generator $\mathbf{Q}$ are square matrices of order $\mathrm{k}+1$.

We denote

$$
S_{1}=-(\lambda+k \mu) \quad S_{2}=(1-p) k \mu
$$

The matrix $\mathbf{A}_{\mathbf{0 0}}$ is described as

$$
\left(\begin{array}{cccccccc}
-\lambda & \lambda & \mathbf{0} & \mathbf{0} & \ldots & \mathbf{0} & \mathbf{0} & \mathbf{0} \\
\mathbf{0} & \mathbf{S}_{1} & \mathbf{k} \boldsymbol{\mu} & \mathbf{0} & \ldots & \mathbf{0} & \mathbf{0} & \mathbf{0} \\
\mathbf{0} & \mathbf{0} & \mathbf{S}_{1} & \mathbf{k} \boldsymbol{\mu} & \ldots & \mathbf{0} & \mathbf{0} & \mathbf{0} \\
\mathbf{0} & \mathbf{0} & \mathbf{0} & \mathbf{S}_{1} & \ldots & \mathbf{0} & \mathbf{0} & \mathbf{0} \\
\ldots & \ldots & \ldots & \ldots & \ldots & \ldots & \ldots & \ldots \\
\mathbf{0} & \mathbf{0} & \mathbf{0} & \mathbf{0} & \ldots & \mathbf{S}_{1} & \mathbf{k \mu} & \mathbf{0} \\
\mathbf{S}_{2} & \mathbf{0} & \mathbf{0} & \mathbf{0} & \ldots & \mathbf{0} & \mathbf{S}_{1} & \mathbf{p k \mu} \\
\boldsymbol{\alpha} & \mathbf{0} & \mathbf{0} & \mathbf{0} & \ldots & \mathbf{0} & \mathbf{0} & -(\lambda+\alpha) \\
& & & & & & &
\end{array}\right)
$$

The Matrix $\mathbf{A}_{\mathbf{n n - 1}}=\left(\mathbf{a}_{\mathbf{i j}}\right)$ where $\mathbf{a}_{\mathbf{i j}}=\mathbf{n} \boldsymbol{\sigma}$ if $\mathrm{i}=1, \mathrm{j}=2$

$$
=\mathbf{0} \text { otherwise }
$$

The matrix $\mathbf{A}_{\mathbf{n} \mathbf{n}}$ for $\mathrm{n}=1,2,3, \ldots$ as

$$
\left(\begin{array}{cccccccc}
-(\lambda+n \boldsymbol{\sigma}) & \lambda & \mathbf{0} & \mathbf{0} & \ldots & \mathbf{0} & \mathbf{0} & \mathbf{0} \\
\mathbf{0} & \mathbf{S}_{1} & \mathbf{k \mu} & \mathbf{0} & \ldots & \mathbf{0} & \mathbf{0} & \mathbf{0} \\
\mathbf{0} & \mathbf{0} & \mathbf{S}_{1} & \mathbf{k} \boldsymbol{\mu} & \ldots & \mathbf{0} & \mathbf{0} & \mathbf{0} \\
\mathbf{0} & \mathbf{0} & \mathbf{0} & \mathbf{S}_{1} & \ldots & \mathbf{0} & \mathbf{0} & \mathbf{0} \\
\ldots & \ldots & \ldots & \ldots & \ldots & \ldots & \ldots & \ldots \\
\mathbf{0} & \mathbf{0} & \mathbf{0} & \mathbf{0} & \ldots & \mathbf{S}_{1} & \mathbf{k} \boldsymbol{\mu} & \mathbf{0} \\
\mathbf{S}_{2} & \mathbf{0} & \mathbf{0} & \mathbf{0} & \ldots & \mathbf{0} & \mathbf{S}_{1} & \mathbf{p k \mu} \\
\boldsymbol{\alpha} & \mathbf{0} & \mathbf{0} & \mathbf{0} & \ldots & \mathbf{0} & \mathbf{0} & -(\boldsymbol{\lambda}+\boldsymbol{\alpha}) \\
& & & & & & &
\end{array}\right)
$$

The matrix $\quad \mathbf{A}_{\mathbf{n n + 1}}=\mathbf{A}_{\mathbf{0}}=\left(\mathbf{a}_{\mathrm{ij}}\right)$ for $\mathbf{n}=\mathbf{0 , 1}, \mathbf{2}, \ldots$

$$
\text { Where } \quad \begin{aligned}
\mathbf{a}_{\mathrm{ij}} & =\boldsymbol{\lambda} \text { if } \mathrm{i}=\mathrm{j}, \mathrm{i}=2,3,4, \ldots, \mathrm{k}+1 \\
& =\mathbf{0} \text { otherwise }
\end{aligned}
$$

If the capacity of the orbit is finite say M then

The matrix $\mathbf{A}_{\mathbf{M M}}$ is given below

$$
\left(\begin{array}{ccccccc}
-(\lambda+M \sigma) & \lambda & \mathbf{0} & \mathbf{0} & \ldots & \mathbf{0} & \mathbf{0} \\
\mathbf{0} & -\mathbf{k} \boldsymbol{\mu} & \mathbf{k \mu} & \mathbf{0} & \ldots & \mathbf{0} & \mathbf{0} \\
\mathbf{0} & \mathbf{0} & -\mathbf{k \mu} & \mathbf{k \mu} & \ldots & \mathbf{0} & \mathbf{0} \\
\mathbf{0} & \mathbf{0} & \mathbf{0} & -\mathbf{k} \boldsymbol{\mu} & \ldots & \mathbf{0} & \mathbf{0} \\
\ldots & \ldots & \ldots & \ldots & \ldots & \ldots & \ldots \\
(1-\mathbf{p}) \mathbf{k} \mu & \mathbf{0} & \mathbf{0} & \mathbf{0} & \ldots & -\mathbf{k} \boldsymbol{\mu} & \mathbf{p k \mu}
\end{array}\right)
$$

Let $\mathbf{X}$ be a steady-state probability vector of $\mathbf{Q}$ and partitioned as $\mathbf{X}=(\mathrm{x}(0), \mathrm{x}(1), \mathrm{x}(2), \ldots)$ and $\mathbf{X}$ satisfies

$$
\mathrm{XQ}=\mathbf{0}, \mathrm{Xe}=1
$$

(1)

where $x(i)=\left(P_{i 0}, P_{i 1}, P_{i 2}, \ldots, P_{i k}, P_{i k+1}\right)$

In this paper we are applying the Direct Truncation Method to find the Steady state probability vector $\mathbf{X}$. Let $\mathbf{M}$ denote the cutoff point for this truncation method. The steady state probability vector $\mathbf{X}^{(\mathbf{M})}$ is now partitioned as

$$
\mathbf{X}^{(\mathbf{M})}=(\mathrm{x}(0), \mathrm{x}(1), \mathrm{x}(2), \ldots . \mathrm{x}(\mathrm{M}))
$$

which satisfies

$$
\mathbf{X}^{(\mathbf{M})} \mathbf{Q}=\mathbf{0}, \mathbf{X}^{(\mathbf{M})} \mathbf{e}=\mathbf{1},
$$

where $x(i)=\left(P_{i 0}, P_{i 1}, P_{i 2}, \ldots, P_{i k+1}\right) \quad i=0,1,2,3, \ldots, M$.

The above system of equations is solved by exploiting the special structure of the co-efficient matrix. It is solved by GAUSSJORDAN elementary transformation method. Since there is no 
clear cut choice for M, we may start the iterative process by taking, say $M=1$ and increase it until the individual elements of $\mathbf{x}$ do not change significantly. That is, if $\mathrm{M}^{*}$ denotes the truncation point then $\left\|\mathbf{x}^{\mathbf{M}^{*}}(\mathbf{i})-\mathbf{x}^{\mathbf{M}^{*-1}}(\mathbf{i})\right\|_{\infty}<\mathbf{\epsilon}$, where $\mathbf{\epsilon}$ is an infinitesimal quantity.

\subsection{STABILITY CONDITION}

\section{Theorem :}

The inequality $\left(\frac{\lambda}{\mu}+\frac{p \lambda}{\alpha}\right)<1$ is the necessary and sufficient condition for system to be stable.

Proof:

Let $\mathrm{Q}$ be an infinitesimal generator matrix for the queueing system (without retrial)

The stationary probability vector $\mathrm{X}$ satisfying

(2)

$$
X Q=0 \text { and } X e=1
$$

Let $\mathrm{R}$ be the rate matrix and satisfying the equation

$$
\mathbf{A}_{0}+\mathbf{R} \mathbf{A}_{1}+\mathbf{R}^{2} \mathbf{A}_{2}=\mathbf{0}
$$

The system is stable if $\operatorname{sp}(\mathrm{R})<1$

We know that the Matrix $R$ satisfies $\operatorname{sp}(\mathrm{R})<1$ if and only if

$$
\Pi \mathbf{A}_{0} \mathbf{e}<\Pi_{\mathbf{2}} \mathbf{e}
$$

where $\boldsymbol{\Pi}=\left(\pi_{1}, \ldots, \pi_{\mathrm{k}+1}\right)$ and satisfies

$$
\Pi A=0 \text { and } \Pi e=1
$$

and

$$
\mathbf{A}=\mathbf{A}_{0}+\mathbf{A}_{1}+\mathbf{A}_{2}
$$

Here $\mathbf{A}_{\mathbf{0}}, \mathbf{A}_{\mathbf{1}}$ and $\mathbf{A}_{\mathbf{2}}$ are square matrices of order $\mathbf{k}$ and

$\mathbf{A}_{\mathbf{0}}=\lambda \mathbf{I}, \mathbf{I}$ the identity matrix of order $\mathrm{k}+1$

The matrix $\mathbf{A}_{1}$ is given below

$$
\left(\begin{array}{cccccc}
\mathbf{s}_{1} & \mathbf{k} \mu & \mathbf{0} & \ldots & \mathbf{0} & \mathbf{0} \\
\mathbf{0} & \mathbf{s}_{1} & \mathbf{k} \mu & \ldots & \mathbf{0} & \mathbf{0} \\
\mathbf{0} & \mathbf{0} & \mathbf{s}_{1} & \ldots & \mathbf{0} & \mathbf{0} \\
\cdots & \cdots & \ldots & \cdots & \cdots & \cdots \\
\mathbf{0} & \mathbf{0} & \mathbf{0} & \ldots & \mathbf{s}_{1} & \mathbf{k \mu} \\
\boldsymbol{\alpha} & \mathbf{0} & \mathbf{0} & \ldots & \mathbf{0} & -(\boldsymbol{\lambda}+\boldsymbol{\alpha})
\end{array}\right)
$$

The matrix $\mathbf{A}_{\mathbf{2}}$ is described as

$$
\left(\begin{array}{cccccc}
0 & 0 & \mathbf{0} & \cdots & \mathbf{0} & \mathbf{0} \\
\mathbf{0} & \mathbf{0} & \mathbf{0} & \cdots & \mathbf{0} & \mathbf{0} \\
\mathbf{0} & \mathbf{0} & \mathbf{0} & \cdots & \mathbf{0} & \mathbf{0} \\
\cdots & \cdots & \cdots & \cdots & \cdots & \cdots \\
(1-p) k \mu & \mathbf{0} & \mathbf{0} & \cdots & \mathbf{0} & \mathbf{k \mu} \\
\mathbf{0} & \mathbf{0} & \mathbf{0} & \cdots & \mathbf{0} & \mathbf{0}
\end{array}\right.
$$$$
\hat{\gamma}
$$

By substituting $\mathbf{A}_{0}, \mathbf{A}_{1}, \mathbf{A}_{2}$ in equations (4), (5) and (6), we get $\left(\frac{\lambda}{\mu}+\frac{p \lambda}{\alpha}\right)<1$. The inequality $\left(\frac{\lambda}{\mu}+\frac{p \lambda}{\alpha}\right)<1$ is also a sufficient condition for the retrial queueing system to be stable. Let $\mathrm{Q}_{\mathrm{n}}$ be the number of customers in the orbit after the departure of $n^{\text {th }}$ customer from the service station. We first prove the embedded Markov chain $\left\{\mathbf{Q}_{\mathbf{n},} \mathbf{n} \geq \mathbf{0}\right\}$ is ergodic if $\left(\frac{\lambda}{\mu}+\frac{p \lambda}{\alpha}\right)<1 .\left\{\mathbf{Q}_{\mathbf{n}}, \mathbf{n} \geq \mathbf{0}\right\}$ is irreducible and aperiodic. It remains to be proved that $\left\{\mathbf{Q}_{\mathbf{n}}, \mathbf{n} \geq \mathbf{0}\right\}$ is positive recurrent. The irreducible and aperiodic Markov chain $\left\{\mathbf{Q}_{\mathbf{n}}, \mathbf{n} \geq \mathbf{0}\right\}$ is positive recurrent if $\left|\psi_{\mathbf{m}}\right|<\infty$ for all $\mathrm{m}$ and $\lim _{\mathbf{m} \rightarrow \infty} \sup _{\mathbf{m}}<0$, where

$$
\psi_{\mathbf{m}}=\mathbf{E}\left(\left(\mathbf{Q}_{\mathbf{n}+\mathbf{1}}-\mathbf{Q}_{\mathbf{n}}\right) / \mathbf{Q}_{\mathbf{n}}=\mathbf{m}\right) \quad(\mathrm{m}=0,1,2,3,4,5, \ldots)
$$$$
\psi_{\mathbf{m}}=\left(\frac{\lambda}{\mu}+\frac{p \lambda}{\alpha}\right)-\left(\frac{m \sigma}{\lambda+m \sigma}\right)
$$

If $\left(\frac{\lambda}{\mu}+\frac{p \lambda}{\alpha}\right)<1$, then $\left|\psi_{\mathbf{m}}\right|<\infty$ for all $\mathbf{m}$ and

$\lim _{\mathbf{m} \rightarrow \infty} \sup _{\mathbf{m}}<0$. Therefore the embedded Markov chain

$\left\{\mathbf{Q}_{\mathbf{n}}, \mathbf{n} \geq \mathbf{0}\right\}$ is ergodic.

\subsection{SPECIAL CASES}

1. If $K=1$ and $p=0$, then this model becomes the Single server retrial queueing model and our numerical results coincide with the following closed form of Number of customers in the orbit in the steady state [6]

$$
\text { Mean Number of Customers in the orbit }=\frac{\rho(\lambda+\rho \sigma)}{(1-\rho) \sigma}
$$

2. As $\sigma \rightarrow \infty$ and $p=0$, the closed form of number of customers in the orbit tends to length of the queue in standard queueing system with Erlang-k service [4] 


$$
\mathrm{L}_{\mathrm{q}}=\left(\frac{k+1}{2 k}\right)\left(\frac{\rho^{2}}{1-\rho}\right)
$$

For many values of $\lambda, \mu, \mathrm{k}$ and very high values of $\sigma(>10000)$, the above result coincides with our numerical results.

3. If $\mathrm{k}=1, \mathrm{p}=0$ and $\sigma \rightarrow \infty$, then mean number of customers in the orbit coincides with

$$
\mathrm{Lq}=\left(\frac{\rho^{2}}{1-\rho}\right)
$$

\subsection{SYSTEM PERFORMANCE MEASURES}

In this section some important performance measures along with formulas and their qualitative behaviour for this queueing model are studied. Numerical study has been dealt in very large scale to study these measures. Defining

$$
\begin{aligned}
\mathrm{P}(\mathrm{n}, 0)= & \text { Probability that there are } \mathrm{n} \text { customers in } \\
& \text { the orbit and server is free } \\
\mathrm{P}(\mathrm{n}, \mathrm{i})= & \text { Probability that there are } \mathrm{n} \text { customers in } \\
& \text { the orbit, server is busy with customer in } \\
& \text { the } \mathrm{i}^{\text {th }} \text { phase for } \mathrm{i}=1,2,3, \ldots \mathrm{k} .
\end{aligned}
$$

We can find various probabilities for various values of $\lambda, \mu, p, \alpha$, $\mathrm{k}$ and $\sigma$ and the following parameters can be easily studied with these probabilities

\section{a. The probability mass function of Server state}

Let $S(t)$ be the random variable which represents the phase in which customer is getting service at time $t$.

Prob $($ The server is idle $)=\sum_{i=0}^{\infty} p(i, 0)$

Prob (The server busy with customer in the $\mathrm{j}^{\text {th }}$ phase)

$$
=\sum_{i=0}^{\infty} p(i, j)
$$

Prob ( The server is in vacation $)=\sum_{i=0}^{\infty} p(i, k+1)$

b. The probability mass function number of customers in the orbit

Let $X(t)$ be the random variable representing the number of customers in the orbit.
Prob (No customers in the orbit) $=\sum_{j=0}^{k+1} p(0, j)$

Prob $\left(\mathrm{i}\right.$ customers in the orbit) $=\sum_{j=0}^{k+1} p(i, j)$

c. The Mean number of customers in the orbit

$$
\mathrm{MNCO}=\sum_{i=0}^{\infty} i\left(\sum_{j=0}^{k+1} p(i, j)\right)
$$

d. The probability that the orbiting customer is blocked

$$
\text { Blocking Probability }=\sum_{i=1}^{\infty} \sum_{j=1}^{k+1} p(i, j)
$$

e. The probability that an arriving customer enter into service immediately

$$
\text { PSI } \quad=\sum_{i=0}^{\infty} p(i, 0)
$$

\subsection{NUMERICAL STUDY}

$$
\begin{array}{ll}
\text { MNCO } & \text { : Mean Number of Customers in the Orbit } \\
\mathrm{P}_{0} & \text { : Probability that the server is idle } \\
\mathrm{P}_{1} & \text { : Probability that the server is busy } \\
\mathrm{P}_{2} & \text { : Probability that the server is in vacation }
\end{array}
$$

Table 1 and Table 2 show the effect of retrial rate $\sigma$ over the system. As $\sigma$ increases, mean number of customers in the orbit decreases and this model becomes classical queueing system with Bernoulli vacation if $\sigma>\mathbf{5 0 0 0}$. Probabilities $P_{\mathbf{0}}$, $P_{1}$ and $P_{2}$ are independent of $\sigma$

Table 1: System Measures for $\lambda=4 \mu=10 \alpha=100$ $\mathbf{p}=\mathbf{0 . 5}$

\begin{tabular}{|c|c|c|c|c|c|}
\hline$\sigma$ & Ocut & MNCO & $\mathrm{P}_{0}$ & $\mathrm{P}_{1}$ & $\mathrm{P}_{2}$ \\
\hline 10 & 12 & 0.4703 & 0.58 & 0.4 & 0.02 \\
\hline 20 & 11 & 0.3255 & 0.58 & 0.4 & 0.02 \\
\hline 30 & 11 & 0.2772 & 0.58 & 0.4 & 0.02 \\
\hline 40 & 11 & 0.2531 & 0.58 & 0.4 & 0.02 \\
\hline 50 & 11 & 0.2386 & 0.58 & 0.4 & 0.02 \\
\hline 60 & 11 & 0.229 & 0.58 & 0.4 & 0.02 \\
\hline 70 & 11 & 0.2221 & 0.58 & 0.4 & 0.02 \\
\hline 80 & 11 & 0.2169 & 0.58 & 0.4 & 0.02 \\
\hline 90 & 11 & 0.2129 & 0.58 & 0.4 & 0.02 \\
\hline
\end{tabular}




\begin{tabular}{|l|l|l|l|l|l|}
100 & 11 & 0.2097 & 0.58 & 0.4 & 0.02 \\
\hline 200 & 11 & 0.1952 & 0.58 & 0.4 & 0.02 \\
\hline 300 & 10 & 0.1903 & 0.58 & 0.4 & 0.02 \\
\hline 400 & 10 & 0.1879 & 0.58 & 0.4 & 0.02 \\
\hline 500 & 10 & 0.1865 & 0.58 & 0.4 & 0.02 \\
\hline 600 & 10 & 0.1855 & 0.58 & 0.4 & 0.02 \\
\hline 700 & 10 & 0.1848 & 0.58 & 0.4 & 0.02 \\
\hline 800 & 10 & 0.1843 & 0.58 & 0.4 & 0.02 \\
\hline 900 & 10 & 0.1839 & 0.58 & 0.4 & 0.02 \\
\hline 1000 & 10 & 0.1836 & 0.58 & 0.4 & 0.02 \\
\hline 2000 & 10 & 0.1821 & 0.58 & 0.4 & 0.02 \\
\hline 3000 & 10 & 0.1817 & 0.58 & 0.4 & 0.02 \\
\hline 4000 & 10 & 0.1814 & 0.58 & 0.4 & 0.02 \\
\hline 5000 & 10 & 0.1813 & 0.58 & 0.4 & 0.02 \\
\hline 6000 & 10 & 0.1812 & 0.58 & 0.4 & 0.02 \\
\hline 7000 & 10 & 0.1811 & 0.58 & 0.4 & 0.02 \\
\hline
\end{tabular}

Table 2 : System Measures for $\lambda=8 \quad \mu=10 \quad \alpha=100$ $\mathbf{p}=\mathbf{0 . 5}$

\begin{tabular}{|c|c|c|c|c|c|}
\hline$\sigma$ & Ocut & MNCO & $\mathrm{P}_{0}$ & $\mathrm{P}_{1}$ & $\mathrm{P}_{2}$ \\
\hline 10 & 62 & 6.82 & 0.16 & 0.8 & 0.04 \\
\hline 20 & 56 & 4.72 & 0.16 & 0.8 & 0.04 \\
\hline 30 & 54 & 4.02 & 0.16 & 0.8 & 0.04 \\
\hline 40 & 53 & 3.67 & 0.16 & 0.8 & 0.04 \\
\hline 50 & 52 & 3.46 & 0.16 & 0.8 & 0.04 \\
\hline 60 & 51 & 3.32 & 0.16 & 0.8 & 0.04 \\
\hline 70 & 51 & 3.22 & 0.16 & 0.8 & 0.04 \\
\hline 80 & 51 & 3.145 & 0.16 & 0.8 & 0.04 \\
\hline 90 & 51 & 3.0866 & 0.16 & 0.8 & 0.04 \\
\hline 100 & 50 & 3.04 & 0.16 & 0.8 & 0.04 \\
\hline 200 & 50 & 2.83 & 0.16 & 0.8 & 0.04 \\
\hline 300 & 49 & 2.76 & 0.16 & 0.8 & 0.04 \\
\hline 400 & 49 & 2.725 & 0.16 & 0.8 & 0.04 \\
\hline 500 & 49 & 2.704 & 0.16 & 0.8 & 0.04 \\
\hline 600 & 49 & 2.69 & 0.16 & 0.8 & 0.04 \\
\hline 700 & 49 & 2.68 & 0.16 & 0.8 & 0.04 \\
\hline 800 & 49 & 2.6725 & 0.16 & 0.8 & 0.04 \\
\hline 900 & 49 & 2.6666 & 0.16 & 0.8 & 0.04 \\
\hline 1000 & 49 & 2.662 & 0.16 & 0.8 & 0.04 \\
\hline 2000 & 49 & 2.641 & 0.16 & 0.8 & 0.04 \\
\hline 3000 & 49 & 2.634 & 0.16 & 0.8 & 0.04 \\
\hline 4000 & 49 & 2.6305 & 0.16 & 0.8 & 0.04 \\
\hline 5000 & 49 & 2.6284 & 0.16 & 0.8 & 0.04 \\
\hline 6000 & 49 & 2.627 & 0.16 & 0.8 & 0.04 \\
\hline 7000 & 49 & 2.626 & 0.16 & 0.8 & 0.04 \\
\hline
\end{tabular}

Table 3 shows the effect of number of phases (k) over the system. As $k$ increases, mean number of customers in the orbit decreases. Probabilities $\mathbf{P}_{0}, \mathbf{P}_{1}$ and $\mathrm{P}_{2}$ are independent of $\mathbf{k}$.

Table 4 shows the effect of probability of going for vacation (p) over the system. As $p$ increases, mean number of customers in the orbit increases.

Table 3 : System Measures for $\lambda=5 \quad \mu=10 \quad \alpha=100$ $\mathrm{p}=\mathbf{0 . 5} \quad \boldsymbol{\sigma}=\mathbf{1 0 0}$

\begin{tabular}{|c|c|c|c|c|c|}
\hline $\mathrm{k}$ & Ocut & MNCO & $P_{0}$ & $P_{1}$ & $P_{2}$ \\
\hline 1 & 20 & 0.6105 & 0.475 & 0.5 & 0.025 \\
\hline 2 & 16 & 0.4789 & 0.475 & 0.5 & 0.025 \\
\hline 3 & 15 & 0.4351 & 0.475 & 0.5 & 0.025 \\
\hline 4 & 14 & 0.4132 & 0.475 & 0.5 & 0.025 \\
\hline 5 & 14 & 0.4 & 0.475 & 0.5 & 0.025 \\
\hline 6 & 14 & 0.3912 & 0.475 & 0.5 & 0.025 \\
\hline 7 & 14 & 0.385 & 0.475 & 0.5 & 0.025 \\
\hline 8 & 14 & 0.3803 & 0.475 & 0.5 & 0.025 \\
\hline 9 & 13 & 0.3766 & 0.475 & 0.5 & 0.025 \\
\hline 10 & 13 & 0.3737 & 0.475 & 0.5 & 0.025 \\
\hline 11 & 13 & 0.3713 & 0.475 & 0.5 & 0.025 \\
\hline 12 & 13 & 0.3693 & 0.475 & 0.5 & 0.025 \\
\hline 13 & 13 & 0.3676 & 0.475 & 0.5 & 0.025 \\
\hline 14 & 13 & 0.3662 & 0.475 & 0.5 & 0.025 \\
\hline 15 & 13 & 0.3649 & 0.475 & 0.5 & 0.025 \\
\hline 16 & 13 & 0.3638 & 0.475 & 0.5 & 0.025 \\
\hline 17 & 13 & 0.3628 & 0.475 & 0.5 & 0.025 \\
\hline 18 & 13 & 0.362 & 0.475 & 0.5 & 0.025 \\
\hline 19 & 13 & 0.3612 & 0.475 & 0.5 & 0.025 \\
\hline 20 & 13 & 0.3605 & 0.475 & 0.5 & 0.025 \\
\hline 21 & 13 & 0.3599 & 0.475 & 0.5 & 0.025 \\
\hline 22 & 13 & 0.3593 & 0.475 & 0.5 & 0.025 \\
\hline 23 & 13 & 0.3588 & 0.475 & 0.5 & 0.025 \\
\hline 24 & 13 & 0.3583 & 0.475 & 0.5 & 0.025 \\
\hline 25 & 13 & 0.3579 & 0.475 & 0.5 & 0.025 \\
\hline 26 & 13 & 0.3575 & 0.475 & 0.5 & 0.025 \\
\hline 27 & 13 & 0.3571 & 0.475 & 0.5 & 0.025 \\
\hline 28 & 13 & 0.3568 & 0.475 & 0.5 & 0.025 \\
\hline 29 & 13 & 0.3564 & 0.475 & 0.5 & 0.025 \\
\hline 30 & 13 & 0.3561 & 0.475 & 0.5 & 0.025 \\
\hline 31 & 13 & 0.3559 & 0.475 & 0.5 & 0.025 \\
\hline 32 & 13 & 0.3556 & 0.475 & 0.5 & 0.025 \\
\hline 33 & 13 & 0.3553 & 0.475 & 0.5 & 0.025 \\
\hline 34 & 13 & 0.3551 & 0.475 & 0.5 & 0.025 \\
\hline 35 & 13 & 0.3549 & 0.475 & 0.5 & 0.025 \\
\hline
\end{tabular}

Table 4 : System Measures for $\lambda=5 \quad \mu=10 \quad \alpha=100$ $\mathbf{k}=\mathbf{5} \quad \boldsymbol{\sigma}=\mathbf{1 0 0}$

\begin{tabular}{|c|c|c|c|c|c|}
\hline$p$ & Ocut & MNCO & $P_{0}$ & $P_{1}$ & $P_{2}$ \\
\hline 0.02 & 13 & 0.3519 & 0.499 & 0.5 & 0.001 \\
\hline 0.04 & 13 & 0.3538 & 0.498 & 0.5 & 0.002 \\
\hline 0.06 & 13 & 0.3557 & 0.497 & 0.5 & 0.003 \\
\hline 0.08 & 13 & 0.3577 & 0.496 & 0.5 & 0.004 \\
\hline
\end{tabular}




\begin{tabular}{|c|c|c|c|c|c|}
\hline 0.1 & 13 & 0.3596 & 0.495 & 0.5 & 0.005 \\
\hline 0.12 & 13 & 0.3615 & 0.494 & 0.5 & 0.006 \\
\hline 0.14 & 13 & 0.3635 & 0.493 & 0.5 & 0.007 \\
\hline 0.16 & 14 & 0.3654 & 0.492 & 0.5 & 0.008 \\
\hline 0.18 & 14 & 0.3674 & 0.491 & 0.5 & 0.009 \\
\hline 0.2 & 14 & 0.3694 & 0.49 & 0.5 & 0.01 \\
\hline 0.22 & 14 & 0.3714 & 0.489 & 0.5 & 0.011 \\
\hline 0.24 & 14 & 0.3734 & 0.488 & 0.5 & 0.012 \\
\hline 0.26 & 14 & 0.3754 & 0.487 & 0.5 & 0.013 \\
\hline 0.28 & 14 & 0.3774 & 0.486 & 0.5 & 0.014 \\
\hline 0.3 & 14 & 0.3794 & 0.485 & 0.5 & 0.015 \\
\hline 0.32 & 14 & 0.3814 & 0.484 & 0.5 & 0.016 \\
\hline 0.34 & 14 & 0.3834 & 0.483 & 0.5 & 0.017 \\
\hline 0.36 & 14 & 0.3855 & 0.482 & 0.5 & 0.018 \\
\hline 0.38 & 14 & 0.3875 & 0.481 & 0.5 & 0.019 \\
\hline 0.4 & 14 & 0.3896 & 0.48 & 0.5 & 0.02 \\
\hline 0.42 & 14 & 0.3916 & 0.479 & 0.5 & 0.021 \\
\hline 0.44 & 14 & 0.3937 & 0.478 & 0.5 & 0.022 \\
\hline 0.46 & 14 & 0.3958 & 0.477 & 0.5 & 0.023 \\
\hline 0.48 & 14 & 0.3979 & 0.476 & 0.5 & 0.024 \\
\hline 0.5 & 14 & 0.4 & 0.475 & 0.5 & 0.025 \\
\hline 0.52 & 14 & 0.4021 & 0.474 & 0.5 & 0.026 \\
\hline 0.54 & 14 & 0.4042 & 0.473 & 0.5 & 0.027 \\
\hline 0.56 & 14 & 0.4064 & 0.472 & 0.5 & 0.028 \\
\hline 0.58 & 14 & 0.4085 & 0.471 & 0.5 & 0.029 \\
\hline 0.6 & 14 & 0.4106 & 0.47 & 0.5 & 0.03 \\
\hline 0.62 & 14 & 0.4128 & 0.469 & 0.5 & 0.031 \\
\hline 0.64 & 14 & 0.415 & 0.468 & 0.5 & 0.032 \\
\hline 0.66 & 14 & 0.4171 & 0.467 & 0.5 & 0.033 \\
\hline 0.68 & 14 & 0.4193 & 0.466 & 0.5 & 0.034 \\
\hline 0.7 & 14 & 0.4215 & 0.465 & 0.5 & 0.035 \\
\hline 0.72 & 14 & 0.4237 & 0.464 & 0.5 & 0.036 \\
\hline 0.74 & 15 & 0.4259 & 0.463 & 0.5 & 0.037 \\
\hline 0.76 & 15 & 0.4281 & 0.462 & 0.5 & 0.038 \\
\hline 0.78 & 15 & 0.4304 & 0.461 & 0.5 & 0.039 \\
\hline 0.8 & 15 & 0.4326 & 0.46 & 0.5 & 0.04 \\
\hline
\end{tabular}

MODEL II

\subsection{MODEL DESCRIPTION}

Consider a single server retrial queueing system with exhaustive type vacation in which customers arrive in a Poisson process with arrival rate $\lambda$. These customers are identified as primary calls. Let $\mathrm{k}$ be the number of phases in the service station. Assume that the service time has Erlang-k distribution with service rate $\mathrm{k} \mu$ for each phase. The vacation rate follows an exponential distribution with parameter $\boldsymbol{\alpha}$. If the server is free at the time of a primary or repeated call arrival, then this arriving call begins to be served immediately and leaves the system after completion of the service. The server goes for vacation compulsorily after servicing to all customers and the system becomes empty. This type of vacation in queueing theory is called exhaustive service type single vacation. The single vacation means after completion of vacation period he can again go for vacation once again after servicing atleast one customer. The server may return from the vacation at any time and is independent of number of customers in the system. We assume that the services in all phases are independent and identical and only one customer at a time is in the service mechanism. If the server is free at the time of a primary call arrival, the arriving call begins to be served in Phase 1 immediately by the server then progresses through the remaining phases and must complete the last phase and leave the system before the next customer enters the first phase. If the server is busy or on vacation, then the arriving customer goes to orbit and becomes a source of repeated calls. This pool of sources of repeated calls may be viewed as a sort of queue. Every such source produces a Poisson process of repeated calls with intensity $\boldsymbol{\sigma}$. If an incoming repeated call finds the server free, it is served in the same manner and leaves the system after service, while the source which produced this repeated call disappears. Otherwise, the system state does not change. We assume that the access from the orbit to the service facility governed by classical retrial rate policy. The input flow of primary calls, interval between repetitions and service time in phases are mutually independent.

\subsection{MATRIX GEOMETRIC SOLUTIONS}

The random process is described as

$\{\langle\mathrm{N}(\mathrm{t}), \mathrm{S}(\mathrm{t})\rangle / \mathrm{N}(\mathrm{t})=0,1,2,3 \ldots ; \mathrm{S}(\mathrm{t})=0,1,2,3, \ldots, \mathrm{k}, \mathrm{k}+1\}$

$\mathrm{S}(\mathrm{t})=0$ if the server being idle

$\mathrm{S}(\mathrm{t})=\mathrm{i}$ for server being busy with the customer in the

$\mathrm{i}^{\text {th }}$ phase for $\mathrm{i}=1,2,3, \ldots, \mathrm{k}$

$\mathrm{S}(\mathrm{t})=\mathrm{k}+1$ for the server to be on vacation.

The possible state spaces are

$$
\{(\mathbf{i}, \mathbf{j}) / \mathrm{i}=0,1,2,3, \ldots ; \mathrm{j}=0,1,2,3, \ldots, \mathrm{k}, \mathrm{k}+1\}
$$

The matrices $\mathbf{A}_{\mathbf{0 0}}, \mathbf{A}_{\mathbf{0 1}}, \mathbf{A}_{\mathbf{n} \mathbf{n - 1}}, \mathbf{A}_{\mathbf{n} \mathbf{n}}$ and $\mathbf{A}_{\mathbf{n} \mathbf{n} \mathbf{1}}$ for $\mathbf{n}$ $=1,2,3, \ldots$ in the infinitesimal matrix generator $\mathbf{Q}$ are square matrices of order $\mathrm{k}+1$.

The matrix $\mathbf{A}_{\mathbf{0 0}}$ is described as

$\left(\begin{array}{cccccccl}-\lambda & \lambda & \mathbf{0} & \mathbf{0} & \cdots & \mathbf{0} & \mathbf{0} & \mathbf{0} \\ \mathbf{0} & \mathbf{S}_{1} & \mathbf{k} \boldsymbol{\mu} & \mathbf{0} & \cdots & \mathbf{0} & \mathbf{0} & \mathbf{0} \\ \mathbf{0} & \mathbf{0} & \mathbf{S}_{1} & \mathbf{k} \boldsymbol{\mu} & \cdots & \mathbf{0} & \mathbf{0} & \mathbf{0} \\ \mathbf{0} & \mathbf{0} & \mathbf{0} & \mathbf{S}_{1} & \cdots & \mathbf{0} & \mathbf{0} & \mathbf{0} \\ \cdots & \cdots & \cdots & \cdots & \cdots & \cdots & \cdots & \cdots \\ \mathbf{0} & \mathbf{0} & \mathbf{0} & \mathbf{0} & \cdots & \mathbf{S}_{1} & \mathbf{k} \boldsymbol{0} & \mathbf{0} \\ \mathbf{0} & \mathbf{0} & \mathbf{0} & \mathbf{0} & \cdots & \mathbf{0} & \mathbf{S}_{1} & \mathbf{k} \boldsymbol{\mu} \\ \boldsymbol{\alpha} & \mathbf{0} & \mathbf{0} & \mathbf{0} & \cdots & \mathbf{0} & \mathbf{0} & -(\lambda+\boldsymbol{\alpha}) \\ & & & & & & & \end{array}\right)$




$$
\begin{aligned}
\mathbf{A}_{\mathbf{n n - 1}}=\left(\mathbf{a}_{\mathbf{i j}}\right) \quad \text { for } \mathbf{n} & =\mathbf{1 , 2}, \mathbf{3}, \ldots \\
\text { where } \quad \mathbf{a}_{\mathbf{i j}} & =\mathbf{n} \boldsymbol{\sigma} \text { if } \mathrm{i}=1, \mathrm{j}=2 \\
& =\mathbf{0}
\end{aligned}
$$

The matrix $\mathbf{A}_{\mathbf{n} \mathbf{n}}$ for $\mathrm{n}=1,2,3, \ldots$ are

$$
\left(\begin{array}{cccccccc}
-(\lambda+n \boldsymbol{\sigma}) & \lambda & \mathbf{0} & \mathbf{0} & \ldots & \mathbf{0} & \mathbf{0} & \mathbf{0} \\
\mathbf{0} & \mathbf{S}_{1} & \mathbf{k \mu} & \mathbf{0} & \ldots & \mathbf{0} & \mathbf{0} & \mathbf{0} \\
\mathbf{0} & \mathbf{0} & \mathbf{S}_{1} & \mathbf{k \mu} & \ldots & \mathbf{0} & \mathbf{0} & \mathbf{0} \\
\mathbf{0} & \mathbf{0} & \mathbf{0} & \mathbf{S}_{1} & \ldots & \mathbf{0} & \mathbf{0} & \mathbf{0} \\
\ldots & \ldots & \ldots & \ldots & \ldots & \ldots & \ldots & \ldots \\
\mathbf{0} & \mathbf{0} & \mathbf{0} & \mathbf{0} & \ldots & \mathbf{S}_{1} & \mathbf{k} \boldsymbol{\mu} & \mathbf{0} \\
\mathbf{k} \boldsymbol{\alpha} & \mathbf{0} & \mathbf{0} & \mathbf{0} & \ldots & \mathbf{0} & \mathbf{S}_{1} & \mathbf{0} \\
\boldsymbol{\alpha} & \mathbf{0} & \mathbf{0} & \mathbf{0} & \ldots & \mathbf{0} & \mathbf{0} & -(\boldsymbol{\lambda + \alpha})
\end{array}\right)
$$

$A_{n n+1}=A_{0}=\left(a_{i j}\right)$ for $n=0,1,2 \ldots$

where, $\quad \mathbf{a}_{\mathbf{i i}}=\boldsymbol{\lambda}$ if $\mathrm{i}=2,3,4, \ldots, \mathrm{k}+1$

$$
=\mathbf{0} \text { otherwise }
$$

If the capacity of the orbit is finite say $\mathrm{M}$ then

The matrix $\mathrm{A}_{\mathrm{MM}}$ is given below

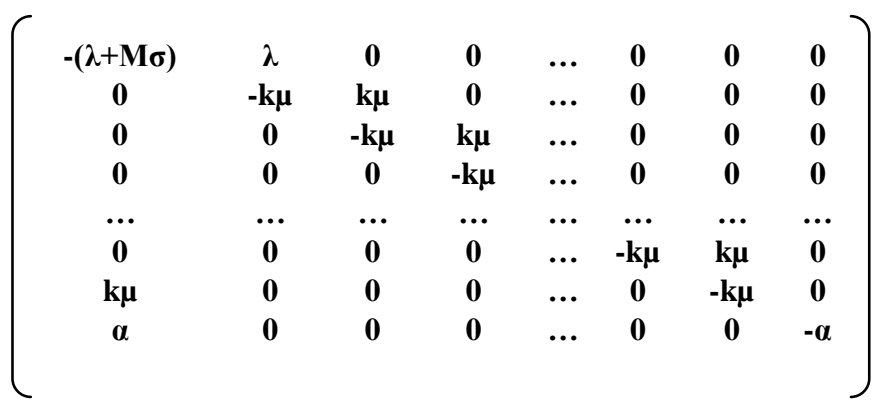

Let $\mathbf{X}$ be a steady-state probability vector of $\mathbf{Q}$ and partitioned as $\mathbf{X}=(\mathrm{x}(0), \mathrm{x}(1), \mathrm{x}(2), \ldots)$ and $\mathbf{X}$ satisfies

$$
\mathrm{XQ}=\mathbf{0}, \mathrm{Xe}=1
$$

where $\mathrm{x}(\mathrm{i})=\left(\mathrm{P}_{\mathrm{i} 0}, \mathrm{P}_{\mathrm{i} 1}, \mathrm{P}_{\mathrm{i} 2}, \ldots \ldots \ldots . . . \mathrm{P}_{\mathrm{ik}}, \mathrm{P}_{\mathrm{ik}+1}\right)$

In this paper we are applying the Direct Truncation Method to find the Steady state probability vector $\mathbf{X}$. Let $\mathbf{M}$ denote the cutoff point for this truncation method. The steady state probability vector $\mathbf{X}^{(\mathbf{M})}$ is now partitioned as

$$
\mathbf{X}^{(\mathbf{M})}=(\mathrm{x}(0), \mathrm{x}(1), \mathrm{x}(2), \ldots . . \mathrm{x}(\mathrm{M}))
$$

which satisfies

$$
\mathbf{X}^{(\mathbf{M})} \mathbf{Q}=\mathbf{0}, \mathbf{X}^{(\mathbf{M})} \mathbf{e}=\mathbf{1},
$$

where $x(i)=\left(P_{i 0}, P_{i 1}, P_{i 2}, \ldots, P_{i k+1}\right) \quad i=0,1,2,3, \ldots, M$.

The above system of equations is solved by exploiting the special structure of the co-efficient matrix. It is solved by GAUSS-JORDAN elementary transformation method. Since there is no clear cut choice for $\mathrm{M}$, we may start the iterative process by taking, say $M=1$ and increase it until the individual elements of $\mathbf{x}$ do not change significantly. That is, if $\mathbf{M}^{*}$ denotes the truncation point then

$\left\|\mathbf{x}^{\mathbf{M}^{*}}(\mathbf{i})-\mathbf{x}^{\mathbf{M}^{*}-\mathbf{1}}(\mathbf{i})\right\|_{\infty}<\mathbf{\epsilon}$, where $\mathbf{\epsilon}$ is an infinitesimal quantity.

\subsection{STABILITY CONDITION}

Theorem :

The inequality $\left(\frac{\lambda}{\mu}\right)<1$ is the necessary and sufficient condition for system to be stable.

Proof:

Let $\mathrm{Q}$ be an infinitesimal generator matrix for the queueing system (without retrial)

The stationary probability vector $\mathrm{X}$ satisfying

$$
X Q=0 \text { and } X e=1
$$

Let $\mathrm{R}$ be the rate matrix and satisfying the equation

$$
\mathbf{A}_{0}+\mathbf{R} \mathbf{A}_{1}+\mathbf{R}^{2} \mathbf{A}_{2}=\mathbf{0}
$$

The system is stable if $\operatorname{sp}(\mathrm{R})<1$

We know that the Matrix $R$ satisfies $\operatorname{sp}(\mathrm{R})<1$ if and only if

$$
\Pi_{\mathbf{A}} \mathbf{e}<\boldsymbol{\Pi A}_{2} \mathbf{e}
$$

where $\boldsymbol{\Pi}=\left(\pi_{1}, \ldots, \pi_{\mathrm{k})}\right.$ and satisfies

$$
\Pi A=0 \text { and } \Pi e=1
$$

and $\quad \mathbf{A}=\mathbf{A}_{0}+\mathbf{A}_{1}+\mathbf{A}_{2}$

Here $\mathbf{A}_{0}, \mathbf{A}_{\mathbf{1}}$ and $\mathbf{A}_{2}$ are square matrices of order $\mathbf{k}$ and

$\mathbf{A}_{\mathbf{0}}=\boldsymbol{\lambda} \mathbf{I}, \mathbf{I}$ is corresponding identity matrix

The matrix $\mathbf{A}_{\mathbf{1}}$ is given below

$$
\left(\begin{array}{ccccccc}
\mathbf{S}_{1} & \mathbf{k} \mu & \mathbf{0} & \cdots & \mathbf{0} & \mathbf{0} & \mathbf{0} \\
\mathbf{0} & \mathbf{S}_{1} & \mathbf{k} \boldsymbol{\mu} & \cdots & \mathbf{0} & \mathbf{0} & \mathbf{0} \\
\mathbf{0} & \mathbf{0} & \mathbf{S}_{1} & \cdots & \mathbf{0} & \mathbf{0} & \mathbf{0} \\
\cdots & \cdots & \cdots & \cdots & \cdots & \cdots & \mathbf{0} \\
\mathbf{0} & \mathbf{0} & \mathbf{0} & \cdots & \mathbf{S}_{1} & \mathbf{k} \boldsymbol{\mu} & \mathbf{0} \\
\mathbf{0} & \mathbf{0} & \mathbf{0} & \cdots & \mathbf{0} & \mathbf{S}_{1} & \mathbf{0} \\
\boldsymbol{\alpha} & \mathbf{0} & \mathbf{0} & \cdots & \mathbf{0} & \mathbf{0} & -(\boldsymbol{\lambda}+\boldsymbol{\alpha})
\end{array}\right)
$$

The matrix $\mathbf{A}_{2}$ is described as

$$
\left(\begin{array}{ccccccc}
\mathbf{0} & \mathbf{0} & \mathbf{0} & \cdots & \mathbf{0} & \mathbf{0} & \mathbf{0} \\
\mathbf{0} & \mathbf{0} & \mathbf{0} & \cdots & \mathbf{0} & \mathbf{0} & \mathbf{0} \\
\mathbf{0} & \mathbf{0} & \mathbf{0} & \cdots & \mathbf{0} & \mathbf{0} & \mathbf{0} \\
\cdots & \cdots & \ldots & \cdots & \cdots & \cdots & \cdots \\
\mathbf{0} & \mathbf{0} & \mathbf{0} & \cdots & \mathbf{0} & \mathbf{0} & \mathbf{0} \\
\mathbf{k \mu} & \mathbf{0} & \mathbf{0} & \cdots & \mathbf{0} & \mathbf{0} & \mathbf{0} \\
\mathbf{0} & \mathbf{0} & \mathbf{0} & \cdots & \mathbf{0} & \mathbf{0} & \mathbf{0}
\end{array}\right)
$$


By substituting $\mathbf{A}_{\mathbf{0}}, \mathbf{A}_{\mathbf{1}}, \mathbf{A}_{\mathbf{2}}$ in equations (10), (11) and (12) ,we get $\left(\frac{\lambda}{\mu}\right)<1$. The inequality $\left(\frac{\lambda}{\mu}\right)<1$ is also a sufficient condition for the retrial queueing system to be stable. Let $\mathrm{Q}_{\mathrm{n}}$ be the number of customers in the orbit after the departure of $n^{\text {th }}$ customer from the service station. We first prove the embedded Markov chain $\left\{\mathbf{Q}_{\mathbf{n}}, \quad \mathbf{n} \geq \mathbf{0}\right\}$ is ergodic if $\left(\frac{\lambda}{\mu}\right)<1 .\left\{\mathbf{Q}_{\mathbf{n}}, \mathbf{n} \geq \mathbf{0}\right\}$ is irreducible and aperiodic. It remains to be proved that $\left\{\mathbf{Q}_{\mathbf{n}}, \mathbf{n} \geq \mathbf{0}\right\}$ is positive recurrent. The irreducible and aperiodic Markov chain $\left\{\mathbf{Q}_{\mathbf{n}}, \mathbf{n} \geq \mathbf{0}\right\}$ is positive recurrent if $\boldsymbol{\psi}_{\mathbf{m}} \mid<\infty$ for all $\mathrm{m}$ and $\lim _{\mathbf{m} \rightarrow \infty} \sup \psi_{\mathbf{m}}<0$, where

$$
\begin{aligned}
& \boldsymbol{\psi}_{\mathbf{m}}=\mathbf{E}\left(\left(\mathbf{Q}_{\mathbf{n}+\mathbf{1}}-\mathbf{Q}_{\mathbf{n}}\right) / \mathbf{Q}_{\mathbf{n}}=\mathbf{m}\right), \mathrm{m}=0,1,2,3,4,5, \ldots \\
& \boldsymbol{\psi}_{\mathbf{m}}=\left(\frac{\lambda}{\mu}\right)-\left(\frac{m \sigma}{\lambda+m \sigma}\right)
\end{aligned}
$$

If $\left(\frac{\lambda}{\mu}\right)<1$, then $\left|\psi_{\mathbf{m}}\right|<\infty$ for all $\mathbf{m}$ and $\lim _{\mathbf{m} \rightarrow \infty} \sup \psi_{\mathbf{m}}<$ 0

Therefore the embedded Markov chain $\left\{\mathbf{Q}_{\mathbf{n}}, \mathbf{n} \geq \mathbf{0}\right\}$ is ergodic.

\subsection{SYSTEM PERFORMANCE MEASURES}

In this section some important performance measures along with formulas and their qualitative behaviour for this queueing model are studied. Numerical study has been dealt in very large scale to study these measures. We can find various probabilities for various values of $\lambda, \mu, \alpha, k$, and $\sigma$. The formulas for system measures which are discussed in section $\mathbf{2 . 5}$ hold for this model also.

\subsection{NUMERICAL STUDY}

MNCO : Mean Number of Customers in the Orbit

$\mathrm{P}_{0} \quad$ : Probability that the server is idle

$\mathrm{P}_{1} \quad$ : Probability that the server is busy

$\mathrm{P}_{2} \quad$ : Probability that the server is in vacation

Tables 5 and Table 6 show the effect of retrial rate $\sigma$ over the system. As $\sigma$ increases, mean number of customers in the orbit decreases and this model becomes classical queuing system with Exhaustive vacation if $\sigma>\mathbf{5 0 0 0}$.

Table 5 : System Measures for $\lambda=4 \mu=10 \quad \alpha=100 \quad k=5$

\begin{tabular}{|c|c|c|c|c|c|}
\hline 20 & 11 & 0.3018 & 0.5787 & 0.4000 & 0.0213 \\
\hline 30 & 11 & 0.2553 & 0.5779 & 0.4000 & 0.0221 \\
\hline 40 & 10 & 0.2319 & 0.5774 & 0.4000 & 0.0226 \\
\hline 50 & 10 & 0.2179 & 0.5772 & 0.4000 & 0.0228 \\
\hline 60 & 10 & 0.2085 & 0.5770 & 0.4000 & 0.0230 \\
\hline 70 & 10 & 0.2018 & 0.5768 & 0.4000 & 0.0232 \\
\hline 80 & 10 & 0.1968 & 0.5767 & 0.4000 & 0.0233 \\
\hline 90 & 10 & 0.1929 & 0.5767 & 0.4000 & 0.0233 \\
\hline 100 & 10 & 0.1898 & 0.5766 & 0.4000 & 0.0234 \\
\hline 200 & 10 & 0.1757 & 0.5763 & 0.4000 & 0.0237 \\
\hline 300 & 10 & 0.1710 & 0.5762 & 0.4000 & 0.0238 \\
\hline 400 & 10 & 0.1686 & 0.5762 & 0.4000 & 0.0238 \\
\hline 500 & 10 & 0.1672 & 0.5762 & 0.4000 & 0.0238 \\
\hline 600 & 10 & 0.1663 & 0.5761 & 0.4000 & 0.0239 \\
\hline 700 & 10 & 0.1656 & 0.5761 & 0.4000 & 0.0239 \\
\hline 800 & 10 & 0.1651 & 0.5761 & 0.4000 & 0.0239 \\
\hline 900 & 10 & 0.1647 & 0.5761 & 0.4000 & 0.0239 \\
\hline 1000 & 10 & 0.1644 & 0.5761 & 0.4000 & 0.0239 \\
\hline 2000 & 10 & 0.1630 & 0.5761 & 0.4000 & 0.0239 \\
\hline 3000 & 10 & 0.1625 & 0.5761 & 0.4000 & 0.0239 \\
\hline 4000 & 10 & 0.1623 & 0.5761 & 0.4000 & 0.0239 \\
\hline 5000 & 10 & 0.1622 & 0.5760 & 0.4000 & 0.0240 \\
\hline 6000 & 10 & 0.1621 & 0.5760 & 0.4000 & 0.0240 \\
\hline 7000 & 10 & 0.1620 & 0.5760 & 0.4000 & 0.0240 \\
\hline 8000 & 10 & 0.1619 & 0.5760 & 0.4000 & 0.0240 \\
\hline
\end{tabular}

\begin{tabular}{|c|c|c|c|c|r|}
\hline$\sigma$ & Ocut & MNCO & $P_{0}$ & $P_{1}$ & $P_{2}$ \\
\hline 10 & 12 & 0.4405 & 0.5811 & 0.4000 & 0.0189 \\
\hline
\end{tabular}

Table 6 : System Measures for $\lambda=8 \quad \mu=10 \quad \alpha=100$ $\mathbf{k}=\mathbf{5}$

\begin{tabular}{|c|c|c|c|c|r|}
\hline$\sigma$ & Ocut & MNCO & $\mathrm{P}_{0}$ & $\mathrm{P}_{1}$ & $\mathrm{P}_{2}$ \\
\hline 10 & 49 & 5.1337 & 0.1969 & 0.8000 & 0.0031 \\
\hline 20 & 44 & 3.5368 & 0.1930 & 0.8000 & 0.0070 \\
\hline 30 & 43 & 3.0026 & 0.1908 & 0.8000 & 0.0092 \\
\hline 40 & 42 & 2.7347 & 0.1895 & 0.8000 & 0.0105 \\
\hline 50 & 41 & 2.5737 & 0.1886 & 0.8000 & 0.0114 \\
\hline
\end{tabular}




\begin{tabular}{|l|l|l|l|l|l|}
60 & 41 & 2.4662 & 0.1879 & 0.8000 & 0.0121 \\
\hline 70 & 40 & 2.3893 & 0.1874 & 0.8000 & 0.0126 \\
\hline 80 & 40 & 2.3316 & 0.1871 & 0.8000 & 0.0129 \\
\hline 90 & 40 & 2.2867 & 0.1868 & 0.8000 & 0.0132 \\
\hline 100 & 40 & 2.2508 & 0.1865 & 0.8000 & 0.0135 \\
\hline 100 & 40 & 2.2508 & 0.1865 & 0.8000 & 0.0135 \\
\hline 200 & 39 & 2.0888 & 0.1854 & 0.8000 & 0.0146 \\
\hline 300 & 39 & 2.0347 & 0.1849 & 0.8000 & 0.0151 \\
\hline 400 & 39 & 2.0076 & 0.1847 & 0.8000 & 0.0153 \\
\hline 500 & 39 & 1.9914 & 0.1846 & 0.8000 & 0.0154 \\
\hline 600 & 39 & 1.9805 & 0.1845 & 0.8000 & 0.0155 \\
\hline 700 & 39 & 1.9728 & 0.1845 & 0.8000 & 0.0155 \\
\hline 800 & 39 & 1.9670 & 0.1844 & 0.8000 & 0.0156 \\
\hline 900 & 39 & 1.9625 & 0.1844 & 0.8000 & 0.0156 \\
\hline 1000 & 39 & 1.9589 & 0.1844 & 0.8000 & 0.0156 \\
\hline 1000 & 39 & 1.9589 & 0.1844 & 0.8000 & 0.0156 \\
\hline 2000 & 39 & 1.9426 & 0.1842 & 0.8000 & 0.0158 \\
\hline 3000 & 39 & 1.9372 & 0.1842 & 0.8000 & 0.0158 \\
\hline 4000 & 39 & 1.9345 & 0.1842 & 0.8000 & 0.0158 \\
\hline 5000 & 39 & 1.9328 & 0.1841 & 0.8000 & 0.0159 \\
\hline 6000 & 39 & 1.9318 & 0.1841 & 0.8000 & 0.0159 \\
\hline 7000 & 39 & 1.9310 & 0.1841 & 0.8000 & 0.0159 \\
\hline 8000 & 39 & 1.9304 & 0.1841 & 0.8000 & 0.0159 \\
\hline 9000 & 39 & 1.9300 & 0.1841 & 0.8000 & 0.0159 \\
\hline 10000 & 39 & 1.9296 & 0.1841 & 0.8000 & 0.0159 \\
\hline & & & & & \\
\hline
\end{tabular}

Table 7 shows the effect of number of phases $(k)$ over the system. As $k$ increases, mean number of customers in the orbit decreases.

Table 8 shows the effect of vacation rate $(\alpha)$ over the system. As $\alpha$ increase, mean number of customers in the orbit decreases.

Table 7 : System Measures for $\lambda=5 \mu=10$ $\alpha=100 \quad \sigma=100$

\begin{tabular}{|c|c|c|c|c|c|}
\hline $\mathrm{k}$ & O_cut & MNCO & P1 & P0 & P2 \\
\hline 1 & 21 & 1.0190 & 0.4827 & 0.5000 & 0.0173 \\
\hline 2 & 18 & 0.8935 & 0.4831 & 0.5000 & 0.0169 \\
\hline 3 & 16 & 0.8517 & 0.4833 & 0.5000 & 0.0167 \\
\hline 4 & 16 & 0.8308 & 0.4834 & 0.5000 & 0.0166 \\
\hline 5 & 15 & 0.8182 & 0.4834 & 0.5000 & 0.0166 \\
\hline 6 & 15 & 0.8099 & 0.4835 & 0.5000 & 0.0165 \\
\hline 7 & 15 & 0.8039 & 0.4835 & 0.5000 & 0.0165 \\
\hline 8 & 15 & 0.7994 & 0.4835 & 0.5000 & 0.0165 \\
\hline 9 & 15 & 0.7959 & 0.4835 & 0.5000 & 0.0165 \\
\hline 10 & 15 & 0.7931 & 0.4835 & 0.5000 & 0.0165 \\
\hline
\end{tabular}

\begin{tabular}{|l|l|l|l|l|l|}
11 & 15 & 0.7908 & 0.4835 & 0.5000 & 0.0165 \\
\hline 12 & 15 & 0.7889 & 0.4835 & 0.5000 & 0.0165 \\
\hline 13 & 15 & 0.7873 & 0.4836 & 0.5000 & 0.0164 \\
\hline 14 & 14 & 0.7859 & 0.4836 & 0.5000 & 0.0164 \\
\hline 15 & 14 & 0.7847 & 0.4836 & 0.5000 & 0.0164 \\
\hline 16 & 14 & 0.7837 & 0.4836 & 0.5000 & 0.0164 \\
\hline 17 & 14 & 0.7828 & 0.4836 & 0.5000 & 0.0164 \\
\hline 18 & 14 & 0.7820 & 0.4836 & 0.5000 & 0.0164 \\
\hline 19 & 14 & 0.7812 & 0.4836 & 0.5000 & 0.0164 \\
\hline 20 & 14 & 0.7806 & 0.4836 & 0.5000 & 0.0164 \\
\hline 21 & 14 & 0.7800 & 0.4836 & 0.5000 & 0.0164 \\
\hline 22 & 14 & 0.7794 & 0.4836 & 0.5000 & 0.0164 \\
\hline 23 & 14 & 0.7789 & 0.4836 & 0.5000 & 0.0164 \\
\hline 24 & 14 & 0.7785 & 0.4836 & 0.5000 & 0.0164 \\
\hline 25 & 14 & 0.7780 & 0.4836 & 0.5000 & 0.0164 \\
\hline 26 & 14 & 0.7777 & 0.4836 & 0.5000 & 0.0164 \\
\hline 27 & 14 & 0.7773 & 0.4836 & 0.5000 & 0.0164 \\
\hline 28 & 14 & 0.7770 & 0.4836 & 0.5000 & 0.0164 \\
\hline 29 & 14 & 0.7767 & 0.4836 & 0.5000 & 0.0164 \\
\hline 30 & 14 & 0.7764 & 0.4836 & 0.5000 & 0.0164 \\
\hline 31 & 14 & 0.7761 & 0.4836 & 0.5000 & 0.0164 \\
\hline 32 & 14 & 0.7758 & 0.4836 & 0.5000 & 0.0164 \\
\hline 33 & 14 & 0.7756 & 0.4836 & 0.5000 & 0.0164 \\
\hline 34 & 14 & 0.7754 & 0.4836 & 0.5000 & 0.0164 \\
\hline 35 & 14 & 0.7752 & 0.4836 & 0.5000 & 0.0164 \\
\hline 36 & 14 & 0.7750 & 0.4836 & 0.5000 & 0.0164 \\
\hline 37 & 14 & 0.7748 & 0.4836 & 0.5000 & 0.0164 \\
\hline 38 & 14 & 0.7746 & 0.4836 & 0.5000 & 0.0164 \\
\hline 39 & 14 & 0.7744 & 0.4836 & 0.5000 & 0.0164 \\
\hline 40 & 14 & 0.7743 & 0.4836 & 0.5000 & 0.0164 \\
\hline & & & & & \\
\hline 14 & & & \\
\hline & & &
\end{tabular}

Table 8 : System Measures for $\lambda=5 \mu=10$ $\mathbf{k}=\mathbf{5} \quad \sigma=100$

\begin{tabular}{|c|c|c|c|c|r|}
\hline$\alpha$ & Ocut & MNCO & $P_{0}$ & $P_{1}$ & $P_{2}$ \\
\hline 10 & 15 & 0.5736 & 0.2968 & 0.5000 & 0.2032 \\
\hline 20 & 13 & 0.4181 & 0.3866 & 0.5000 & 0.1134 \\
\hline 30 & 13 & 0.3837 & 0.4222 & 0.5000 & 0.0778 \\
\hline 40 & 13 & 0.3706 & 0.4410 & 0.5000 & 0.0590 \\
\hline 50 & 13 & 0.3642 & 0.4526 & 0.5000 & 0.0474 \\
\hline 60 & 13 & 0.3606 & 0.4603 & 0.5000 & 0.0397 \\
\hline 70 & 13 & 0.3583 & 0.4659 & 0.5000 & 0.0341 \\
\hline 80 & 13 & 0.3567 & 0.4701 & 0.5000 & 0.0299 \\
\hline
\end{tabular}




\begin{tabular}{|c|c|c|c|c|c|}
\hline 90 & 13 & 0.3556 & 0.4734 & 0.5000 & 0.0266 \\
\hline 100 & 13 & 0.3548 & 0.4761 & 0.5000 & 0.0239 \\
\hline 100 & 13 & 0.3548 & 0.4761 & 0.5000 & 0.0239 \\
\hline 200 & 13 & 0.3518 & 0.4880 & 0.5000 & 0.0120 \\
\hline 300 & 13 & 0.3511 & 0.4920 & 0.5000 & 0.0080 \\
\hline 400 & 13 & 0.3507 & 0.4940 & 0.5000 & 0.0060 \\
\hline 500 & 13 & 0.3506 & 0.4952 & 0.5000 & 0.0048 \\
\hline 600 & 13 & 0.3505 & 0.4960 & 0.5000 & 0.0040 \\
\hline 700 & 13 & 0.3504 & 0.4966 & 0.5000 & 0.0034 \\
\hline 800 & 13 & 0.3503 & 0.4970 & 0.5000 & 0.0030 \\
\hline 900 & 13 & 0.3503 & 0.4973 & 0.5000 & 0.0027 \\
\hline 1000 & 13 & 0.3503 & 0.4976 & 0.5000 & 0.0024 \\
\hline 1000 & 13 & 0.3503 & 0.4976 & 0.5000 & 0.0024 \\
\hline 2000 & 13 & 0.3501 & 0.4988 & 0.5000 & 0.0012 \\
\hline 3000 & 13 & 0.3501 & 0.4992 & 0.5000 & 0.0008 \\
\hline 4000 & 13 & 0.3501 & 0.4994 & 0.5000 & 0.0006 \\
\hline 5000 & 13 & 0.3500 & 0.4995 & 0.5000 & 0.0005 \\
\hline 6000 & 13 & 0.3500 & 0.4996 & 0.5000 & 0.0004 \\
\hline 7000 & 13 & 0.3500 & 0.4997 & 0.5000 & 0.0003 \\
\hline 8000 & 13 & 0.3500 & 0.4997 & 0.5000 & 0.0003 \\
\hline 9000 & 13 & 0.3500 & 0.4997 & 0.5000 & 0.0003 \\
\hline 10000 & 13 & 0.3500 & 0.4998 & 0.5000 & 0.0002 \\
\hline
\end{tabular}

\section{REFERENCES}

[1]. Artalejo. J.R, A queueing system with returning customers and waiting line. Operations Research Letters, 1995, 17, 191-199.

[2]. Artalejo. J.R, A classified bibliography of research on retrial queues: Progress in 1990-1999. Top, 1995b, 7, 187-211.

[3]. B.T. Doshi, Queueing systems with vacations-a survey, Queueing Systems 1 (1986) 29-66.

[4]. B.T. Doshi, Conditional and unconditional distributions for $\mathrm{M} / \mathrm{G} / 1$ type queues with server vacation, Queueing Systems 7 (1990) 229-252.

[5]. Falin. G.I, A survey of retrial queues. Queueing Systems, 1990, 7,127-167.

[6]. Falin. G.I, J.G.C. Templeton, Retrial Queues. Chapman and Hall, London, 1997

[7]. Keilson. J, L.D. Servi, Oscillating random walk models for GI/G/1 vacation systems with Bernoulli schedules, Journal of Applied Probability 23 (1986),pp 790-802

[8]. Marcel F. Neuts, Matrix Geometric Solutions in Stochastic Models an algorithmic approach. 